\title{
The ENT Run Through Pilot: a questionnaire survey of 23 trainees
}

\author{
Elliot Heward ${ }^{1}$ and B. Nirmal Kumar ${ }^{1}$ \\ ${ }^{1}$ Wrightington Wigan and Leigh NHS Foundation Trust
}

June 5, 2020

\begin{abstract}
* ENT run through posts are extremely competitive requiring a median core surgical interview rank of $27 *$ Holding additional degrees and transitioning directly from foundation training may improve applicant success * Prior exposure to ENT is variable in successful ST1 applicants * The pilot has been successful in attracting candidates by providing geographical stability and encouraging early engagement with ENT UK * These results will enable development of the pilot programme and provide valuable information for those applying to an ENT run through post
\end{abstract}

Title : The ENT Run Through Pilot: a questionnaire survey of 23 trainees

Keywords : Education, Otolaryngology, Surveys and Questionnaires, Pilot Projects

Key Points :

- ENT run through posts are extremely competitive requiring a median core surgical interview rank of 27

- Holding additional degrees and transitioning directly from foundation training may improve applicant success

- Prior exposure to ENT is variable in successful ST1 applicants

- The pilot has been successful in attracting candidates by providing geographical stability and encouraging early engagement with ENT UK

- These results will enable development of the pilot programme and provide valuable information for those applying to an ENT run through post

\section{Ethical Considerations :}

Ethical approval was not required.

\section{Background :}

The Ear Nose and Throat (ENT) specialty is known to be underrepresented in the undergraduate curriculum and competition for ST3 posts fell to 1.63 applicants per post in 2016.1,2 Core surgical training was seen as a contributing factor, defocusing and demotivating ENT trainees. The ENT run through programme, introduced in 2018, aimed to improve early recruitment to the specialty by allowing trainees to begin specialist training at ST1. In addition, the run through pilot aimed to provide geographical training stability, competition free transition to ST3, early engagement with ENT UK and refocused ENT training in the early years. 3 Candidate selection occurred at the core surgical interview and is a highly competitive process with 1870 and 1896 applicants in 2018 and 2019 respectively for all surgical specialities.4,5

This study aims to understand what makes a successful interview applicant and the experience of the run through trainees' during the ST1 and ST2 years. The General Medical Council requested evaluation of the programme which this study goes someway to address. 


\section{Materials and Methods :}

A questionnaire survey was sent to all current ENT ST1 and ST2 run through trainees in England on $6^{\text {th }}$ November 2019. The survey had three aims: to identify the background of successful candidates, to determine the satisfaction of the run through trainees and investigate progression to ST3. The questionnaire results were recorded on google forms online.

\section{Results}

Of the 26 run through trainees in England 23 responded (12:ST1, 11:ST2). The educational background of the trainees found 12 attended private and 11 state run secondary school. The most frequent year of graduation was 2016, with the largest number of trainees graduating from The University of Birmingham. Seventeen trainees held additional degrees and 4 were in an alternative training programme prior to starting ST1 (Figure 1). The majority ( $\mathrm{n}=11$ ) of trainees moved straight from foundation training into a run through post with 8 and 4 trainees having a 1 year and 2 year gap respectively. The median amount of ENT exposure (including foundation training) prior to starting ST1 was 4 months (Range: 0-17 months) (Figure 2).

The trainees most commonly decided to pursue a career in ENT on the year of the interview or the year preceding (Figure 3). The median ranking at the core surgical interview was 27 (range: 3-174). In total 91\% ranked a non-run through post. $87 \%$ of those surveyed stated geographical stability was a factor in applying for the run through post.

During the run through pilot $48 \%$ of trainees were contacted by their regional trainee run through lead, of those $45 \%$ found this helpful. Overall, trainees will spend a median of 6 months (range 0-16 months) in both a secondary and tertiary ENT centre, with 10 trainees only experiencing one of these care settings. Additionally, 91\% of trainees have 2 allied specialty placements during their ST1 and ST2 years. The majority of the trainees thought that being on the run-through pilot had increased their ENT trainer engagement which was not the case for non-ENT trainers (Figure 4). Nineteen trainees were members of ENT UK at the time of survey.

Of the 11 surveyed ST2s 9 felt equipped to become an ST3, 3 planned to attend the 2020 national selection and 10 the ST3 bootcamp. The trainees attending ENT national selection had the aim of changing region.

\section{Discussion}

Synopsis of key/new findings

Understanding what makes a successful interview candidate has for some time interested the medical community.6 As the ENT ST3 competition ratio increases (2.57 applicants per post in 2019) junior trainees interested in securing a national training number $(\mathrm{NTN})$ will seek to gain a ST1 run through post.5 In addition, the run through programme will likely further increase the competition ratio at ST3 by reducing overall available posts. This questionnaire demonstrates that schooling, be it private or state, does not affect interview success. Holding an additional degree and transitioning directly from foundation programme seems to increase the likelihood of interview success. Although candidates graduated from 12 different universities a large proportion came from the University of Birmingham (Figure 1). It could be proposed that undergraduates at this institution had a greater exposure to ENT or that training better prepared them for the core interview process.

The pilot currently allows candidates to gain an ENT NTN by a non-ENT selection process. A specialtyspecific selection process would be able to deliver a comparable interview to ST3 recruitment and assess candidates' interest and engagement with ENT. This would standardise recruitment to ENT NTN posts and encourage applications from only those with a true interest in the specialty. This study demonstrates that many current run through trainees had little exposure of ENT prior to application (Figure 2). As a result, it may prevent immediate transfer from foundation training and there is no doubt that duplication of the interview process would increase consultant time required. 
Exposure to ENT certainly does not preclude candidate success (Figure 2). Nine of the trainees had less than 4 months ENT exposure prior to gaining an NTN. Some may argue that this is insufficient exposure to determine a true interest in the specialty and could lead to a higher dissatisfaction and dropout rate. In addition, it is interesting that the majority of trainees decided to pursue a career in ENT during the interview year or 1 year previous (Figure 3). This suggests that the introduction of the run through pilot may have influenced the speciality decision of junior trainees attending the core surgical interview. Results show that the pilot has succeeded by attracting candidates with geographical training stability and the majority of trainees are engaged with ENT UK.

Trainees should have access to a regional trainee run through lead. The questionnaire results demonstrate that less than half of trainees had contact with the lead and those who did only $45 \%$ found this useful. Regional leads may find it difficult to guide and advise trainees when there is little information available and the pilot programme is in its infancy. Furthermore, exposure to secondary and tertiary ENT services seems to be variable amongst the run through trainees. The majority of trainees have exposure to 2 allied specialities which would achieve maximum points in this domain in the 2020 ST3 portfolio marking scheme. Standardisation of placements is required to provide exposure to both secondary and tertiary ENT and allied specialities prior to ST3.

Trainer engagement is vitally important in helping to motivate and fulfil the potential of trainees. The perceived increase in ENT trainer engagement further solidifies the role of the run through programme in ENT training. However, this may come at the cost of reduced engagement with allied speciality trainers who may have a reduced motivation to train those whose speciality interest has already been decided (Figure 4).

Of the ST2 trainees the majority felt prepared to start ST3 and would engage with the ST3 boot camp. It was interesting that $27 \%$ of ST2s intended to attend the ST3 interview in 2020 so to change region. This slightly contradicts the benefit of improved geographical stability with the run through programme.

Strengths of the study

This study had an excellent uptake of the questionnaire with $88 \%$ of the current run through trainees responding in full. This provides a very good representation of the current run through cohort. The total number of trainees was calculated from the number of run through jobs advertised. It is not possible to say whether those who did not respond chose not to, the post was not filled or is now vacant.

Comparisons with other studies

This is the first study to investigate the background and progress of ENT run through trainees. Manjaly and Radford surveyed successful ENT ST3 applicants in 2012.6 They also found that trainees who held additional degrees and spent less time out of training were more likely to be successful at interview. Furthermore, they observed that trainees had a range of ENT exposure (6-30 months) prior to application which mirrors the findings of the current study.6

Clinical applicability of the study

This nationwide ENT run through questionnaire allows an insight into the background and progress of the trainees who were successful in attaining a run through post. The pilot has been successful in attracting candidates by providing geographical stability and removing the need for further interview at ST3. An additional benefit of the run through programme has been to improve ENT trainer engagement which is critical at this stage of training. Changes should be made to improve contact between the regional run through lead and their trainees and to ensure standardisation of placements. These results will enable development of the pilot programme and provide valuable information for those applying to an ENT run through post.

\section{References}

1. Powell J, Cooles FAH, Carrie S, Paleri V. Is undergraduate medical education working for ENT surgery? A survey of UK medical school graduates. J Laryngol Otol 2011;125:896-905. 
2. Green R, Steven R, Richard, Haddow K. Declining applications to surgical specialist training. RCS Bull. 2017;90:142-144.

3. Health Education England. Run Through Pilot in Otolaryngology. https://www.nwpgmd.nhs.uk/sites/default/files/Run\%20Through\%20Pilot\%20in\%20Otolaryngology\%20v2.pdf. Accessed $28^{\text {th }}$ May 2020.

4. Arwyn-Jones J, Bhalla S, Acharya V, Beegun I, Awad Z, Tolley N. Specialty Showcase Days: Can Specialist Careers Workshops Improve The Consideration Of ENT For Medical Students? Adv Med Educ Pract 2019;10:877-884.

5. Health Education England. Specialty Recruitment Competition Ratios 2019. https://specialtytraining.hee.nhs.uk/Portals/1/Competition\%20Ratios\%202019_1.pdf. Accessed $28^{\text {th }}$ May 2020.

6. Manjaly J, Radford P. Who is succeeding at ENT ST3 England National Selection? Ann R Coll Surg Engl (suppl) 2014;96:1-5.

\section{Figure Legends}

Figure 1. ENT run through trainees by undergraduate medical school university

Figure 2. ENT experience prior to starting ENT ST1 post

Figure 3. Decision to pursue a career in ENT prior to ST1 interview

Figure 4. Improved trainer engagement for ENT run through trainees: ENT versus Non ENT trainers

\section{Data Sharing}

The data that support the findings of this study are available from the corresponding author upon reasonable request.

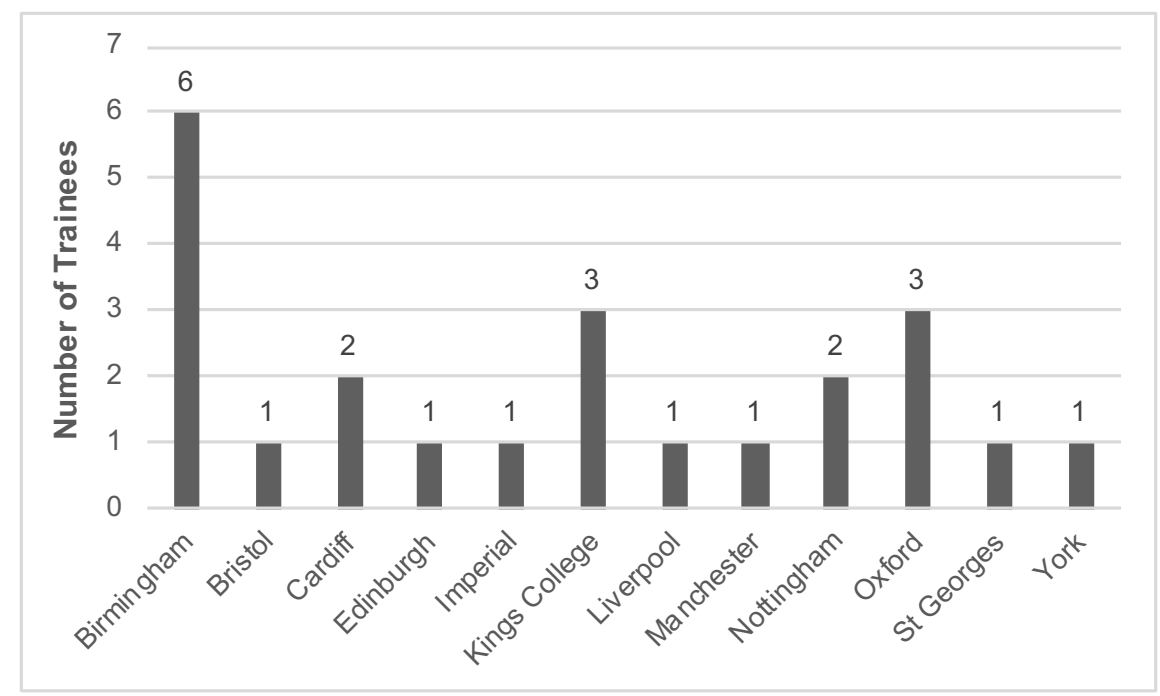



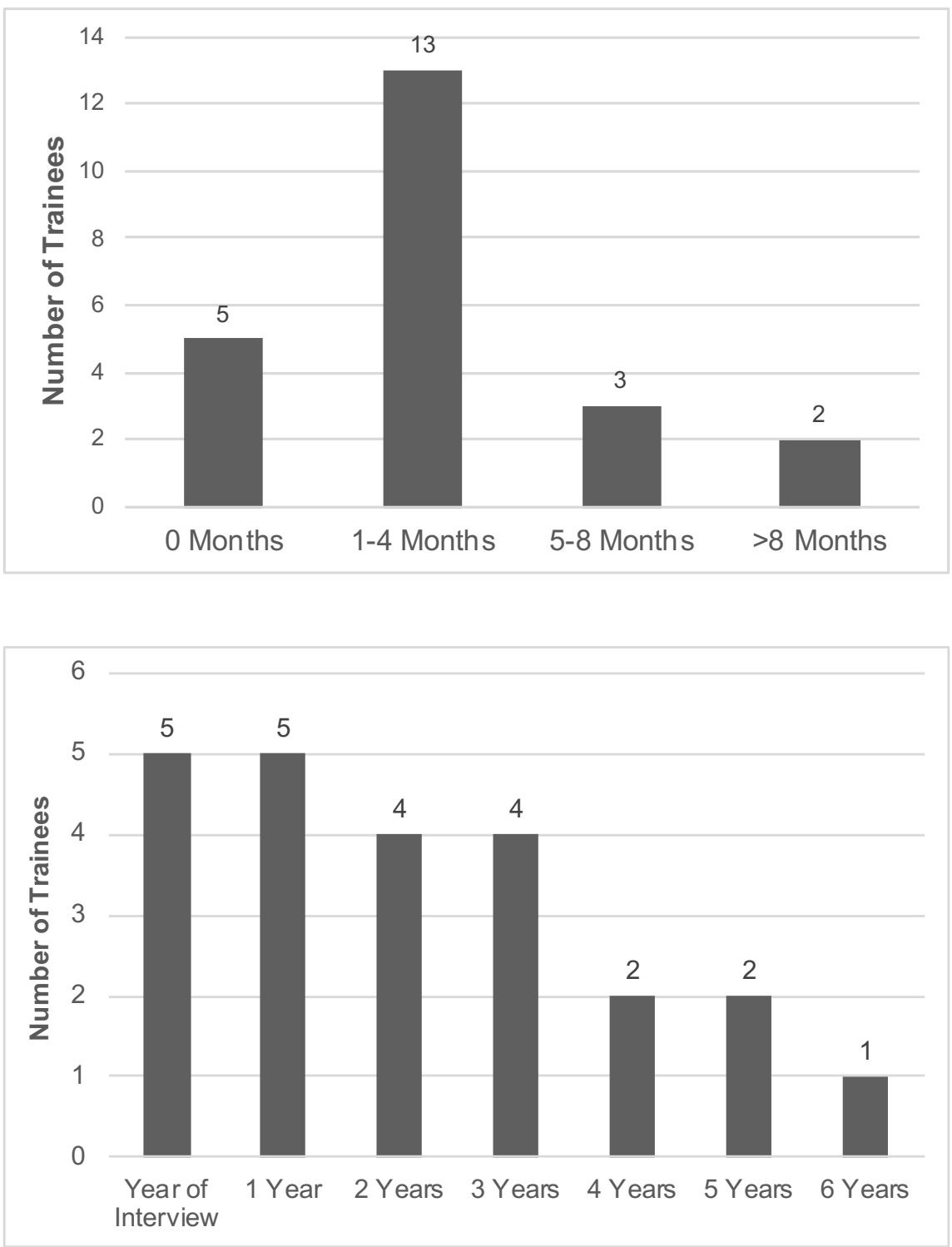


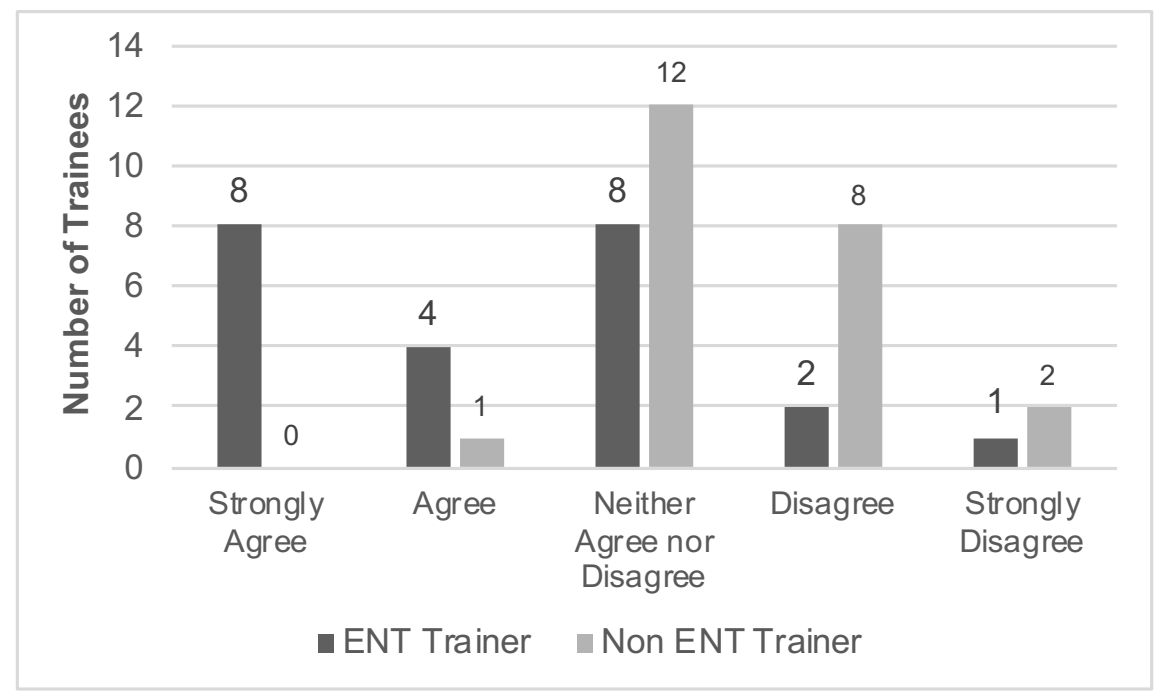

